Running head: RELIABILITY OF POLYSUBSTANCE USE ASSESSMENT

Word Count: 3,879

Figures: 1

Tables: 3

\title{
Test-retest reliability of a new assessment to detect detailed temporal patterns of polysubstance use
}

Nicole D. Fitzgerald ${ }^{\mathrm{a}^{*} \dagger}$, Yiyang Liu ${ }^{\mathrm{a} \dagger}$, Anna Wang ${ }^{\mathrm{a}}$, Catherine W. Striley ${ }^{\mathrm{a}}$, Barry Setlow ${ }^{\mathrm{b}}$, Lori Knackstedt $^{\mathrm{c}}$, Linda B. Cottler ${ }^{\mathrm{a}}$

${ }^{\text {a }}$ Department of Epidemiology, Colleges of Medicine and Public Health \& Health Professions, University of Florida, Gainesville, Florida, USA

${ }^{\mathrm{b}}$ Department of Psychiatry, College of Medicine, University of Florida, Gainesville, Florida, USA

${ }^{c}$ Department of Psychology, College of Liberal Arts and Sciences, University of Florida, Gainesville, Florida, USA

*Corresponding author: Nicole D. Fitzgerald, Department of Epidemiology, Colleges of Medicine and Public Health \& Health Professions, University of Florida, 2004 Mowry Drive, PO Box 100231, Gainesville, Florida 32611 (nfitzgerald@ufl.edu)

$\dagger$ These authors contributed equally to this work and share first authorship. 
Acknowledgements: This research was supported by the National Institute on Drug Abuse of the National Institutes of Health under Award Numbers R21DA045140 (MPIs: Cottler, Knackstedt) and T32DA035167 (PI: Cottler). The funding source had no role in the study design, collection, analysis, interpretation of data, writing of the manuscript, nor the decision to submit the present research. 


\begin{abstract}
Objective: While polysubstance use is highly prevalent among people who use drugs, the field lacks a reliable assessment that can detect detailed temporal patterns of polysubstance use. This study assessed the test-retest reliability of the newly developed Polysubstance Use-Temporal Patterns Section (PSU-TPS).

Methods: Participants who used cocaine plus alcohol and/or marijuana at least once in the past 30 days ( $n=48)$ were interviewed at baseline and approximately seven days later (retest) using the Substance Abuse Module (SAM) and the PSU-TPS. Reliability of PSU-TPS measures of quantity, frequency, and duration of polysubstance use was examined using intra-class correlation coefficients (ICCs) and kappa tests.
\end{abstract}

Results: Excellent reliability was observed for frequencies of concurrent polysubstance use patterns in the past 30 days (ICC range: $0.90-0.94$ ) and quantity of alcohol use (ICC=0.83), and fair to good reliability was observed for duration in hours of substance use (ICC range: $0.52-$ $0.73)$.

Conclusion: Detailed information regarding cocaine, alcohol, and marijuana polysubstance use in the past 30 days can be reliably measured with the PSU-TPS. Data on the order and timing of polysubstance use at the hourly level will improve our understanding of the implications of sequential and simultaneous use patterns, which can help inform treatment and prevention efforts.

Keywords: polysubstance use; cocaine; marijuana; alcohol; reliability 


\section{Introduction}

Although polysubstance use, or the use of multiple substances during a specified period of time, is acknowledged as one of the most common substance use behaviors ( Kandel et al., 2017; Cicero et al., 2019; Compton, Valentino, \& DuPont, 2021) and is associated with a wide range of adverse outcomes (Agrawal et al., 2007; Quek et al., 2013; Leeman et al., 2016), it remains an understudied and poorly defined construct (Bailey and Finn, 2020). For example, many studies do not distinguish between concurrent use- - which refers to two or more substances used within a specified period (e.g., past 12 months, past 30 days) — and sequential or simultaneous use (Liu et al., 2018). Sequential use describes which drug was used first, which second, and so on within the same occasion (e.g., within the same 24 hours), while simultaneous use indicates co-administration of two or more substances at the same time or in close temporal proximity (e.g., the use of a mixture of cocaine and heroin, or smoking marijuana while drinking alcohol) (Barrett, Gross, Garand, \& Pihl, 2005; McCabe et al., 2006; Olthuis et al., 2013).

Both concurrent and simultaneous polysubstance use have been found to be highly prevalent among persons who use cocaine. A meta-analysis estimated that, among those who use cocaine, $77 \%$ used alcohol and $64 \%$ used marijuana concurrently with cocaine, while $74 \%$ used alcohol and 38\% used marijuana simultaneously with cocaine (Liu et al., 2018). Measures of simultaneous cocaine polysubstance use are particularly important, as research has shown that drug combinations can impact subjective responses to cocaine (Lukas et al., 1994; McCanceKatz, Kosten, \& Jatlow, 1998) as well as neurobiological outcomes (Meyerhoff et al., 1999; O’Neill, Cardenas, \& Mayerhoff, 2001; Meyerhoff, 2017). Using alcohol or marijuana with cocaine can affect cocaine's metabolic profile (McCance et al., 1998; Pan \& Hedaya, 2000; Reid \& Bornheim, 2001), potentially enhancing its reinforcing effects (Jatlow et al., 1991; Raven, 
Necessary, Danluck, \& Ettenberg, 2000; Hart, Jatlow, Sevarino, \& McCance-Katz, 2000). Given that these substances can interact both metabolically and neurobiologically depending on the timing of their overlapping use, collecting data on concurrent use alone is insufficient for a comprehensive understanding of the etiology and consequences of cocaine polysubstance use patterns.

Despite the importance of considering the timing and order of sequential and simultaneous polysubstance use, few studies have examined the temporal ordering of cocaine use with alcohol and/or marijuana. Of the five studies that have investigated the order and sequencing of simultaneous cocaine and alcohol use (Staines, Magura, Foote, Deluca, \& Kosanke, 2001; Barrett et al., 2005; Barrett, Darredeau, \& Pihl, 2006; Gossop, Manning, \& Ridge, 2006; Macdonald, Mac Intyre, Joordens, Stockwell, \& Martin, 2015), the majority found that alcohol use generally proceeded the use of cocaine on days in which both were used, with the exception of Gossop et al. (2006), who found that a greater proportion of persons who used crack cocaine reported drinking alcohol after using crack than before or during crack use (36\% vs. $21 \%$ and $12 \%$, respectively). Two of these studies also assessed quantity of alcohol and cocaine use (Barrett et al., 2006; Gossop et al., 2006) and found that, on days in which both cocaine and alcohol were used together, participants reported an increase in average consumption of number of drinks (Barrett et al., 2006) and grams of cocaine (Gossop et al., 2006). Those who specifically used crack cocaine, however, reported a decrease in number of average drinks and no significant change in amount of cocaine consumed (Gossop et al., 2006). Only one of these studies also investigated the order and sequencing of simultaneous cocaine and marijuana use: in a sample of university students, Barrett et al. (2006) found that, among those with lifetime co-use of cocaine and marijuana during a single session, $43 \%$ used marijuana 
before cocaine, while $37.5 \%$ used marijuana over multiple sessions with cocaine use. Changes in marijuana use quantity were not examined, however, due to non-standardized marijuana use units.

The studies that examined the order and sequencing of cocaine plus alcohol and/or marijuana use assessed simultaneous use with one of two methods: researchers either asked participants to describe 1) their typical patterns of use without specifying a time period (Macdonald et al., 2015) or 2) the number of days on which they used each substance over a specified time period (Staines et al., 2001; Barrett et al., 2005; Barrett, et al., 2006; Gossop et al., 2006). Most used a version of the Timeline Followback (TLFB) to record days of use of each drug over a 30- or 90-day period. The TLFB was first developed as a self-report measure for retrospective estimates of alcohol use using a calendar format (Sobell et al., 1997; Sobell et al., 1999) and has since been expanded to assess the use of multiple other substances (Robinson, Sobell, Sobell, \& Leo, 2014; Rendina et al., 2015; Martin-Willett et al., 2020; Goldstick et al., 2022). The TLFB has been compared with other clinical measures and biological measures and has demonstrated good reliability and validity (Eyawo et al., 2020; Martin-Willett et al., 2020). In addition to assessing frequency of concurrent and simultaneous use (i.e., days in which substances were used alone or together) and quantity of use on those days with a modified TLFB approach, these studies also examined order of ingestion by asking participants to describe the order in which cocaine was used with either alcohol or marijuana for each day of co-use (Gossop et al., 2006), the most recent day of co-use (Barrett et al., 2006), or their most typical day of use (Staines et al., 2001). However, this method of enumeration of the order of substance use provides limited resolution regarding the duration, timing, and temporal proximity of episodelevel simultaneous use on the specified day (Martin, 2008), and variations in selection of an 
anchor for recall between these studies (each day, most recent day, typical day) make comparisons difficult.

Given the limitations of the current literature, there is a significant need for methods that can assess detailed, temporal patterns of polysubstance use-including quantity, frequency, and duration of use - in order to inform treatment and prevention efforts. As part of a larger study, we developed and tested a new assessment that combines a calendar-based approach with a comprehensive, hour-by-hour examination of within-day temporality for each use pattern to measure concurrent, sequential, and simultaneous polysubstance use in persons who use cocaine. To determine the reliability of this new assessment - the Polysubstance Use-Temporal Patterns Section (PSU-TPS) — we adopted a test-retest study design to examine the reliability of polysubstance use quantity, frequency, and duration measures, and assessed reasons for discrepancies.

\section{Materials and Methods}

\subsection{Sample}

This analysis was based on data collected as part of a NIDA-funded study to identify patterns of human polysubstance use to guide the development of preclinical models (R21DA045140). The test-retest phase of this study was conducted from December 2019 through March 2021. Interviews began in-person before converting to remote interviews over Zoom in March 2020 due to the COVID-19 pandemic. Participants were eligible if they were between 18 to 65 years of age and reported 1) past 30-day cocaine use, 2) at least five occasions of cocaine use within the past 12-months, and 3) past 30-day alcohol and/or marijuana use. Prior to March 2020, participants were recruited via flyers distributed throughout Gainesville, Florida 
and through HealthStreet, the University of Florida Clinical and Translational Science Institute (CTSI)'s community engagement program. Once social distancing guidelines were implemented, recruitment methods were broadened to include online recruitment tools such as ResearchMatch, Consent2Share, and Facebook advertisements. All study protocols were approved by the University of Florida Institutional Review Board (IRB).

\subsection{Assessments}

The PSU-TPS was developed as a calendar-based interview followed by an hour-level examination of typical days of use for each pattern to collect information on the frequency, duration, quantity, preference, and order of the use of cocaine, alcohol, and marijuana. The flow, wordings of the questions, and skipped patterns of PSU-TPS are demonstrated in Figure 1. It begins by displaying a calendar of the past 30 days to determine on how many days each of the three substances were consumed together or separately. For each established pattern (cocaine+alcohol+marijuana [CAM], cocaine+alcohol, no marijuana $[\mathrm{CA}]$, cocaine + marijuana, no alcohol [CM], cocaine [C] only, alcohol [A] only, or marijuana [M] only), participants were asked to select a "typical day" in the past 30 days for their use. For each typical day of use, participants were asked to indicate on a 24-hour timeline (beginning at 6 a.m. and ending at 5 a.m.) when they used each substance. Up to three episodes (hourly blocks of continuous use) were elicited for each substance, and for each episode, participants were asked about the route of administration (except for alcohol) and quantity of each of the substances used. Quantity of

alcohol use was reported in standard drinks. For cocaine and marijuana, participants reported their quantity of use in number, and chose a corresponding unit from a card that included 25 common units (e.g., grams, lines, joints) of substance use. 
The Substance Abuse Module (SAM, earlier known as part of the CIDI-SAM) is a fully structured diagnostic interview that assesses lifetime and past 12-month use and DSM-5 diagnostic criteria for a wide range of psychoactive substances and has demonstrated excellent reliability, as detailed in prior studies (Compton et al., 1996a; Compton et al., 1996b; Cottler et al., 1989; Horton et al., 2000). For the purpose of this analysis, past 12-month DSM-5 cocaine, alcohol, and cannabis use disorders were examined, as well as other variables including age, sex, race/ethnicity, marital status, and education level.

\subsection{Test-retest procedures}

Full interview procedures for both test and retest are shown in Figure 1. The computerized PSU-TPS and SAM were installed on laptop computers and administered at both the baseline and retest interviews by two separate interviewers to minimize bias, with the retest interviewer blinded to baseline responses. At each interview, the interviewer sat with the participant in a private room (pre-COVID)/a password-protected Zoom (post-COVID), read each

of the questions to participants, and recorded responses. For some questions (like those referring to the past 30 day calendar), the interviewer shared their screen with participants to facilitate responses. Retest interviews were conducted approximately one week after baselines. The Discrepancy Interview Protocol (DIP) (Bidaut-Russell et al., 1995; Cottler et al., 1994; Cottler et al., 1997; Cottler et al., 2009) was administered after the retest interview to determine reasons for discrepancies in answers between the baseline and retest for selected PSU-TPS measures (including frequency of CAM, CA, and CM days, start and end times and route of cocaine use on a typical CAM/CA/CM day, and preferred drug use without considering accessibility). For each determined polysubstance use pattern, if the number of days in the past 30 days, duration of hourly use on a typical day, quantity of use on a typical day, or substance of preference differed 
between baseline and retest, participants were asked to explain the discrepancy by choosing one of 12 reasons such as not understanding the question, feeling too embarrassed to answer, or remembering differently between interviews.

All interviewers completed training and certification (provided by LBC and CWS, SAM authors and trainers) prior to administering the interviews. Participants were compensated $\$ 25$ USD after the baseline and \$25 USD after the retest interview for their time and effort, with a \$10 USD bonus for being on time without having to reschedule.

\subsection{Analyses}

To assess test-retest reliability for continuous variables, intra-class correlation coefficients (ICCs) were calculated along with their 95\% confidence intervals (CIs). For categorical data, kappa tests were performed. For both ICCs and kappa values, $\geq 0.75$ represents excellent reliability, values between 0.60 and 0.74 represent good reliability, values between 0.40 and 0.60 represent fair reliability, and values $<0.40$ represent poor reliability (Cicchetti, 1995).

\section{Results}

As shown in Table 1, among the 48 participants enrolled, the mean age of the sample was 30.5 (SD 15.3) and 35.4\% were female. Half identified as non-Hispanic White, $20 \%$ as nonHispanic Black, 20.8\% as Hispanic, 6.3\% as Asian, and 2.1\% as American Indian or Alaska Native. The majority were never married (83.3\%) and had high school or above educational attainment (83.3\%). Thirty-three interviews (68.8\%) were conducted in-person, with $15(31.3 \%)$ over Zoom. All participants reported cocaine and alcohol use in the past 12 months. Among the sample, $52.1 \%$ met the criteria for cocaine use disorder in the past 12 months and $66.7 \%$ for 
alcohol use disorder. All but three participants reported marijuana use in the past 12 months. Among users, $62.2 \%$ met the criteria for cannabis use disorder in the past 12 months.

Table 2 provides data on mean and ICC values on selected numerical variables and cell number and kappa values on selected categorical variables. All three variables representing number of days of use for the three primary drugs of interest (cocaine, alcohol, and marijuana) in the past 30 days showed excellent reliability (ICCs ranged 0.90-0.94). Additionally, the PSUTPS had excellent reliability in capturing the frequency of alcohol only use days (A only, $\mathrm{ICC}=0.94)$, marijuana only use days ( $\mathrm{M}$ only, $\mathrm{ICC}=0.92)$, cocaine, alcohol and marijuana use days $(\mathrm{CAM}, \mathrm{ICC}=0.80)$ and cocaine and marijuana but no alcohol use days $(\mathrm{CM}, \mathrm{ICC}=0.78)$. Fair reliability was found for capturing the frequency of cocaine and alcohol use days (CA, $\mathrm{ICC}=0.47)$ and cocaine only use days $(\mathrm{C}$ only, $\mathrm{ICC}=0.42)$.

Among the six use patterns measured, using CAM on the same day was the most common pattern, with 24 people endorsing at least one CAM day in the past 30 days. Therefore, we estimated the reliability of duration and quantity of use measures on a typical CAM day. Fair to good reliability was found for the duration of use of cocaine, alcohol, and marijuana, respectively (ICC range: $0.52-0.73$ ). The variable representing the quantity of alcohol used on a typical CAM day had excellent reliability $(\mathrm{ICC}=0.83)$. When only examining those who reported the same typical CAM day between baseline and retest $(n=9)$, excellent reliability was observed for both duration of cocaine, alcohol, and marijuana use (ICC range: $0.85-0.98$ ) and quantity of alcohol use $(\mathrm{ICC}=0.94)$. Estimating reliability for reported quantity of cocaine and marijuana use is difficult because participants could report their use in different units. Most reported quantity of cocaine use in grams/milligrams/ounces (44\%) or lines $(37 \%)$. Other units reported included rocks, bumps, and hits. Units reported for marijuana use were more diverse: around one-third 
reported in joints, $20 \%$ in grams/milligrams/ounces, $17 \%$ in blunts, $14 \%$ in hits, and a few in breaths or huffs. Participants could also report their use in different units across the two interviews (e.g., cocaine quantity in grams at baseline and in lines at retest). Of the 24 people endorsing at least one CAM day at both baseline and retest interview, 20 (83.3\%) reported quantity of cocaine use in the same units, and $18(75.0 \%)$ reported quantity of marijuana use in the same units. Among people reporting in the same units, the ICC was 0.26 (95\% CI: -0.18 , $0.61)$ for cocaine use quantity and $0.33(-0.13,0.67)$ for marijuana use quantity. Only $15.0 \%$ (cocaine) and $11.0 \%$ (marijuana) had greater than 1 unit differences in their reported quantity of use between baseline and retest.

For the question asking which drug participants would have used if access were not an issue, kappas were estimated for each reported drug. Fair to good reliability was shown for all variables with cell count $>1$ (kappa range: $0.48-0.67$ ).

Reasons for discrepancies between baseline and retest interviews were also examined. "Situation changed since the first interview" (e.g., participants reported cocaine use during a holiday at baseline, and at retest, the holiday was no longer within the past 30 days and not shown on the calendar) and "not remembering the same information" at each interview were the top reasons contributing to different responses for most of the variables. The start and end times of cocaine use on typical days when different polysubstance use of CAM, CA, or CM was endorsed had the highest discrepancies. For these variables, the respondent selecting a different typical day is another key reason why answers differed between two interviews. Among the 24 participants who reported past 30-day CAM use at both baseline and retest, $9(37.5 \%)$ reported the same typical CAM day at both interviews, while 15 selected a different typical day.

\section{Discussion}


This study examined the test-retest reliability of the newly developed PSU-TPS, an assessment designed to collect temporal data on concurrent, sequential, and simultaneous polysubstance use among persons who use cocaine with alcohol and/or marijuana. This new assessment expand past TLFB methods in ability to evaluate detailed, hour-by-hour polysubstance use patterns, which is significant given that simultaneous and sequential polysubstance use is widespread but understudied. The PSU-TPS was found to have excellent reliability for assessing frequencies of concurrent polysubstance use patterns in the past 30 days, fair to good reliability for the duration of substance use on what participants identified as a typical cocaine-alcohol-marijuana polysubstance use day, and excellent reliability for quantity of alcohol use on typical polysubstance use days.

Similar to a multi-substance TLFB approach (Martin-Willett et al., 2020), the PSU-TPS begins with a calendar of the past 30 days and asks participants to endorse whether they used cocaine, alcohol, and/or marijuana on each day, which results in frequencies of daily polysubstance use patterns. Our reliability estimates for frequency of use in the past 30 days for cocaine, marijuana, and alcohol are similar to those reported by test-retest studies evaluating the reliability of frequency of days of cocaine (Ehrman \& Robbins, 1994; Fals-Stewart et al., 2000; Robinson et al., 2014; Wray, Braciszewski, Zywiak, \& Stout, 2016), marijuana (Fals-Stewart et al., 2000; Norberg, Mackenzie, \& Copeland, 2012; Robinson et al., 2014; Wray et al., 2016), and alcohol (Fals-Stewart et al., 2000; Wray et al., 2016) using a TLFB approach. After collecting data on polysubstance patterns on the past 30-day calendar, our assessment deviates from the traditional TLFB methods. Instead of only measuring the total quantity of use per day, our assessment asks participants to describe the hour-level start and end times for each episode of substance use on each typical day, as well as the quantity of use during each episode. Our 
assessment showed similar reliability estimates to TLFB approaches on mean drinks per day (Fals-Stewart et al., 2000). Since no TLFB approach to our knowledge assesses episode-level duration of substance use, we are unable to compare the reliability of our assessment for durations of use to prior studies.

An important feature of our test-retest study design was the inclusion of a Discrepancy Interview Protocol (DIP) (Cottler et al., 1994) at the end of the retest interview to assess reasons for discrepancies between the baseline and retest responses. The number of reported CAM days, number of CA days, and start and end times for the first cocaine use episode on a typical CAM day had some of the highest numbers of discrepant responses. Almost half of participants with discrepant responses on these items attributed these discrepancies to a changed situation or a different reported typical day between the baseline and follow-up interviews. Because the follow-up interview took place up to 11 days following the baseline interview, participants' calendar view for selecting the frequency of past 30-day use often changed by as much as an entire week between baseline and retest, which likely impacted the test-retest reliability of reporting. Roughly half of the discrepancies in number of CAM and CA days in the past 30 days were only different by one day.

In addition to the impact of the inherent changes in participants' calendar view between baseline and follow-up interviews on test-retest reliability, another challenge included variations in the selection of a typical day of use. We observed relatively lower reliabilities for measures that require participants to select a typical day for that use pattern. For example, the test-retest reliability of duration of substance use episodes was lower than that of frequency of past 30-day use, and it is likely that this variation in choice of a typical CAM day in the past 30 days impacted the reliability of duration reporting. Only a quarter of people selected the same typical 
day for CAM at baseline and retest. Among them, excellent reliability for duration was observed. It is possible that some people had more than one typical pattern of polysubstance use when CAM were all used on the same day, or did not have any typical use pattern, as their use varied day by day. Duration and quantity of alcohol use on a typical CAM day were chosen for reliability estimates due to the majority of participants endorsing at least one CAM day $(n=24)$ compared to CA $(n=14)$ or $\mathrm{CM}(n=5)$ days. Thus, it is possible that the reliability of duration and quantity of alcohol use could also vary for typical days of different polysubstance use patterns. Despite these inherent difficulties, however, acceptable test-retest reliability was observed for duration of substance use episodes across all substances.

An additional challenge was measuring the reliability of the quantity of cocaine and marijuana used during substance use episodes. As is done in the SAM (Cottler et al. 1989), participants in the current study were given the option of reporting quantity of use for each episode in up to 25 different units. This flexibility allows participants to easily report the quantity according to their preferred units, but the variations in reported units limited our ability to examine the test-retest reliability for quantity of cocaine and marijuana used. While one option would have been to examine the reliability of quantity of use stratified by unit type (e.g., reliability among people who reported units in joints, grams, rocks, respectively), the sample size was not sufficiently large for these subgroup analyses. Since analyses were conditional on participants endorsing at least one CAM, CA, or CM day at both baseline and retest, sample sizes were further reduced for reliability estimates of some polysubstance use characteristics. The utility of other methodological approaches for assessing quantity, including the use of a scale estimation procedure with a surrogate substance (Mariani, Brooks, Haney, \& Levin, 2011; Norberg et al., 2011; Tomko et al., 2018) and visual scales (Farris et al., 2014; Cuttler \& 
Spradlin, 2017) should be considered in future research. The issue of drug potency, which is not assessed in the PSU-TPS, also complicates the measurement of quantity for cocaine and marijuana and is an additional limitation. Lastly, though the order of the interview was carefully designed to ensure interview flow and questions on similar topics stayed together, the lack of randomization may have inadvertently caused potential order effects in participants responses.

\subsection{Conclusions}

Our results demonstrate that detailed temporal patterns of concurrent, sequential, and simultaneous polysubstance use involving cocaine, alcohol, and marijuana can be reliably measured with the PSU-TPS. Future research should expand this assessment to detect polysubstance use patterns involving other substances, examine between-day fluctuations in use, explore the utility of other standardized measures of quantity and potency, and validate the assessment against similar tools (e.g., a multi-substance TLFB). The utilization of an hour-level analysis of the timing and order of polysubstance use will improve our understanding of the implications of sequential and simultaneous use patterns when combined with data on drugrelated outcomes, which can help inform treatment and prevention efforts in this population. 


\section{Conflict of Interest}

No conflict declared. 


\section{References}

Agrawal, A., Lynskey, M. T., Madden, P. A., Bucholz, K. K., \& Heath, A. C. (2007). A latent class analysis of illicit drug abuse/dependence: results from the National Epidemiological Survey on Alcohol and Related Conditions. Addiction, 102(1), 94-104.

doi:10.1111/j.1360-0443.2006.01630.x

Bailey, A. J., \& Finn, P. R. (2020). Examining the utility of a general substance use spectrum using latent trait modeling. Drug and Alcohol Dependence, 212, 107998. doi:10.1016/j.drugalcdep.2020.107998

Barrett, S. P., Gross, S. R., Garand, I., \& Pihl, R. O. (2005). Patterns of simultaneous polysubstance use in Canadian rave attendees. Substance Use \& Misuse, 40(9-10),152537. doi:10.1081/JA-200066866

Barrett, S. P., Darredeau, C., \& Pihl, R. O. (2006). Patterns of simultaneous polysubstance use in drug using university students. Human Psychopharmacology: Clinical \& Experimental, 21(4), 255-263.

Bidaut-Russell, M., Reich, W., Cottler, L. B., Robins, L. N., Compton, W. M., \& Mattison, R. E. (1995). The Diagnostic Interview Schedule for Children (PC-DISC v. 3.0): Parents and adolescents suggest reasons for expecting discrepant answers. Journal of Abnormal Child Psychology, 23(5), 641-59. doi:10.1007/BF01447667

Chaves, T. V., Sanchez, Z. M., Ribeiro, L. A., \& Nappo, S. A. (2011). Crack cocaine craving: Behaviors and coping strategies among current and former users. Revista de Saúde Pública, 45(6), 1168-75. doi:10.1590/S0034-89102011005000066

Chen, L. Y., Crum, R. M., Martins, S. S., Kaufmann, C. N., Strain, E. C., \& Mojtabai, R. (2014). Patterns of concurrent substance use among nonmedical ADHD stimulant users: Results 
from the National Survey on Drug Use and Health. Drug and Alcohol Dependence, 142, 86-90. doi:10.1016/j.drugalcdep.2014.05.022

Cicchetti, D. V. (1995). Guidelines, criteria, and rules of thumb for evaluating normed and standardized assessment instruments in psychology. Psychological Assessment, 6(4), 284-90. doi:10.1037/1040-3590.6.4.284

Cicero, T. J., Ellis, M. S., Kasper, Z. A. (2019). Polysubstance use: A broader understanding of substance use during the opioid crisis. American Journal of Public Health, 110(2), 244250. doi:10.2105/AJPH.2019.305412

Compton, W. M., Cottler, L. B., Dorsey, K. B., Spitznagel, E. L., \& Mager, D. E. (1996a). Comparing assessments of DSM-IV substance dependence disorders using CIDI-SAM and SCAN. Drug and Alcohol Dependence, 41(3), 179-87. doi:10.1016/0376$8716(96) 01249-5$

Compton, W. M., Cottler, L. B., Dorsey, K. B., Spitznagel, E. L., \& Mager, D. E. (1996b). Structured and semi-structured assessment of ICD-10 substance dependence disorders: CIDI-SAM vs. SCAN. International Journal of Methods in Psychiatric Research, 6, 285293.

Compton, W. M., Valentino, R. J., DuPont, R. L. (2021). Polysubstance use in the U.S. opioid crisis. Molecular Psychiatry, 26(1), 41-50.

Connor, J. P., Gullo, M. J, White, A., \& Kelly, A. B. (2014). Polysubstance use: diagnostic challenges, patterns of use and health. Current Opinion in Psychiatry, 27(4), 269-75. doi:10.1097/YCO.0000000000000069 
Cottler, L. B., Robins, L. N., Helzer, J.E. (1989). The reliability of the CIDI-SAM: a comprehensive substance abuse interview. British Journal of Addiction, 84(7), 801-14. doi:10.1111/j.1360-0443.1989.tb03060.x

Cottler, L. B., Compton, W. M., Brown, L., \& Shell, A. (1994). The Discrepancy Interview Protocol: A method for evaluating and interpreting discordant survey responses. International Journal of Methods in Psychiatric Research, 4(3), 173-182.

Cottler, L. B., Grant, B. F., Blaine, J., Mavreas, V., Pull, C., Hasin, D., Compton, W. M., RubioStipec, M., \& Mager, D. (1997) Concordance of DSM-IV alcohol and drug use disorder criteria and diagnoses as measured by AUDADIS-ADR, CIDI and SCAN. Drug and Alcohol Dependence, 47(3), 195-205. doi:10.1016/S0376-8716(97)00090-2

Cottler, L. B., Leung, K. S., \& Abdallah, A. B. (2009). Test-re-test reliability of DSM-IV adopted criteria for 3, 4-methylenedioxymethamphetamine (MDMA) abuse and dependence: a cross-national study. Addiction, 104(10), 1679-90. doi:10.1111/j.13600443.2009.02649.x

Cuttler, C., \& Spradlin, A. (2017). Measuring cannabis consumption: Psychometric properties of the Daily Sessions, Frequency, Age of Onset, and Quantity of Cannabis Use Inventory (DFAQ-CU). PLoS One, 12(5), e0178194.

Ehrman, R. N. \& Robbins, S. J. (1994). Reliability and validity of 6-month timeline reports of cocaine and heroin use in a methadone population. Journal of Consulting and Clinical Psychology, 62(4), 843. doi:10.1037/0022-006X.62.4.843

Ellinwood Jr, E. H., Eibergen, R. D., Kilbey, M. M. (1976). Stimulants: interaction with clinically relevant drugs. Annals of the New York Academy of Sciences, 281(1), 393408. doi:10.1111/j.1749-6632.1976.tb27948.x 
Eyawo O., Deng Y. Dziura J., et al. (2020). Validating self-reported unhealthy alcohol use with phosphatidylethanol (PEth) among patients with HIV. Alcoholism, Clinical and Experimental Research, 44(10), 2053-2063. doi:10.1111/acer.14435.

Fals-Stewart, W., O'Farrell, T. J., Freitas, T. T., McFarlin, S. K., \& Rutigliano, P. (2000). The timeline followback reports of psychoactive substance use by drug-abusing patients: psychometric properties. Journal of Consulting and Clinical Psychology, 68(1), 134. doi:10.1037/0022-006X.68.1.134

Farris, S. G., Zvolensky, M. J., Boden, M. T., \& Bonn-Miller, M. O. (2014). Cannabis use expectancies mediate the relation between depressive symptoms and cannabis among cannabis-dependent veterans. Journal of Addiction Medicine, 8(2), 130-136.

Goldstick, J. E., Bonar, E. E., Myers, M., Bohnert, A. S., Walton, M. A., \& Cunningham, R. M. (2022). Within-person predictors of same-day alcohol and nonmedical prescription drug use among youth presenting to an urban emergency department. Journal of Studies on Alcohol and Drugs, 83(1), 85-90.

Gossop, M., Manning, V., \& Ridge, G. (2006). Concurrent use and order of use of cocaine and alcohol: behavioral differences between users of crack cocaine and cocaine powder. Addiction, 101(9), 1292-1298.

Hart, C. L., Jatlow, P., Sevarino, K. A., \& McCance-Katz, E. F. (2000). Comparison of intravenous cocaethylene and cocaine in humans. Psychopharmacology, 149(2), 153-162.

Horton, J., Compton, W., \& Cottler, L. B. (2000). Reliability of substance use disorder diagnoses among African-Americans and Caucasians. Drug and Alcohol Dependence, 57(3), 203-9. doi:10.1016/S0376-8716(99)00050-2 
Jatlow, P., Elsworth, J. D., Bradberry, C. W., Winger, G., Taylor, J. R., Russell, R., et al. (1991). Cocaethylene: A neuropharmacologically active metabolite associated with concurrent cocaine-ethanol ingestion. Life Sciences, 48(18), 1787-1794.

Kandel, D. B., Hu, M. C., Griesler, P., \& Wall, M. (2017). Increases from 2002 to 2015 in prescription opioid overdose deaths in combination with other substances. Drug and Alcohol Dependence, 178, 501-11. doi:10.1016/j.drugalcdep.2017.05.047

Leeman, R. F., Sun, Q., Bogart, D., Beseler, C. L., \& Sofuoglu, M. (2016) Comparisons of cocaine-only, opioid-only, and users of both substances in the National Epidemiologic Survey on Alcohol and Related Conditions (NESARC). Substance Use \& Misuse, 51(5), 553-64. doi:10.3109/10826084.2015.1122063

Liu, Y., Williamson, V., Setlow, B., Cottler, L. B., \& Knackstedt, L. A. (2018). The importance of considering polysubstance use: lessons from cocaine research. Drug and Alcohol Dependence, 192, 16-28. doi:10.1016/j.drugalcdep.2018.07.025

Lukas, S. E., Sholar, M., Kouri, E., Fukuzako, H., \& Mendelson, J. H. (1994). Marihuana smoking increases plasma cocaine levels and subjective reports of euphoria in male volunteers. Pharamcology Biochemistry and Behavior, 48(3), 715-721.

Macdonald, S., MacIntyre, P., Joordens, C., Stockwell, T., \& Martin, G. (2015). Factors related to simultaneous cocaine and alcohol use for clients in treatment. Journal of Alcoholism and Drug Dependence, 3(193), 2.

Mariani, J. J., Brooks, D., Haney, M., \& Levin, F. R. (2011). Quantification and comparison of marijuana smoking practices: Blunts, joints, and pipes. Drug and Alcohol Dependence, 113(2-3), 249-251. 
Martin, C. S. (2008). Timing of alcohol and other drug use. Alcohol Research \& Health, 31(2), 96.

Martin-Willett, R., Helmuth, T., Abraha, M., Bryan, A. D., Hitchcock, L., Lee, K, \& Bidwell, L. C. (2020). Vaalidation of a multisubstance online Timeline Followback assessment. Brain and Behavior, 10(1), e01486.

McCabe, S. E., Cranford, J. A., Morales, M., Young, A. (2006). Simultaneous and concurrent polydrug use of alcohol and prescription drugs: prevalence, correlates, and consequences. Journal of Studies on Alcohol, 67(4), 529-37. doi:10.15288/jsa.2006.67.529

McCance-Katz, E. F., Kosten, T. R., \& Jatlow, P. (1998). Concurrent use of cocaine and alcohol is more potent and potentially more toxic than use of either alone-A multiple-dose study. Biological Psychiatry, 44(4), 250-259.

Meyerhoff, D. J., Bloomer, C., Schuff, N., Ezekiel, F., Norman, D., Clark, W., et al. (1999). Cortical metabolite alterations in abstinent cocaine and cocaine/alcohol-dependent subjects: proton magnetic resonance spectroscopic imaging. Addiction Biology, 4(4), 405-419.

Meyerhoff, D. J. (2017). Functionally relevant brain alterations in polysubstance users: difference to monosubstance users, study challenges, and implications for treatment. In Addictive Substances and Neurological Disease (pp. 217-237). Academic Press.

Norberg, M. M., Mackenzie, J., \& Copeland, J. (2012). Quantifying cannabis use with the Timeline Followback approach: A psychometric evaluation. Drug and Alcohol Dependence, 121(3), 247-52. doi:10.1016/j.drugalcdep.2011.09.007 
Olthuis, J. V., Darredeau, C., \& Barrett, S. P. (2013). Substance use initiation: the role of simultaneous polysubstance use. Drug and Alcohol Review, 32(1), 67-71. doi:10.1111/j.1465-3362.2012.00470.x

O’Neil, J., Cardenas, V. A., \& Meyerhoff, D. J. (2001). Separate and interactive effects of cocaine and alcohol dependence on brain structures and metabolites: quantitative MRI and proton MR spectroscopic imaging. Addiction Biology, 6(4), 347-361.

Pan, W-J., \& Hedaya, M. A. (2000). Cocaine and alcohol interactions in the rat: Effect of cocaine and alcohol pretreatments on cocaine pharmacokinetics and pharmacodynamics. Journal of Pharmaceutical Sciences, 88(12), 1266-1274.

Quek, L. H., Chan, G. C., White, A., Connor, J. P., Baker, P., Saunders, J. B., \& Kelly, A. B. (2013). Concurrent and simultaneous polydrug use: latent class analysis of an Australian nationally representative sample of young adults. Frontiers in Public Health, 1, 61. doi:10.3389/fpubh.2013.00061

Raven, M. A., Necessary, B. D., Danluck, D. A., \& Ettenberg, A. (2000). Comparison of the reinforcing and anxiogenic effects of intravenous cocaine and cocaethylene. Experimental and Clinical Psychopharmacology, 8(1), 117.

Reid, M. J., \& Bornheim, L. M. (2001). Cannabinoid-induced alterations in brain disposition of drugs of abuse. Biochemical Pharmacology, 61(11), 1357-1367.

Rendina, H. J., Moody, R. L., Ventuneac, A., Grov, C., \& Parsons, J. T. (2015). Aggregate and event-level associations between substance use and sexual behavior among gay and bisexual men: comparing retrospective and prospective data. Drug and Alcohol Dependence, 154, 199-207. 
Robinson, S. M., Sobell, L. C., Sobell, M. B., \& Leo, G. I. (2014). Reliability of the Timeline Followback for cocaine, cannabis, and cigarette use. Psychology of Addictive Behaviors, 28(1), 154. doi:10.1037/a0030992

Sobell, L. C., Agrawal, S., \& Sobell, M. B. (1997). Factors affecting agreement between alcohol abusers' and their collaterals' reports. Journal of Studies on Alcohol, 58(4), 405-13. doi:10.15288/jsa.1997.58.405

Sobell, L. C., Agrawal, S., \& Sobell, M. B. (1999). Utility of liver function tests for screening “alcohol abusers" who are not severely dependent on alcohol. Substance Use \& Misuse, 34(12), 1723-32. doi:10.3109/10826089909039424

Staines, G. L., Magura, S., Foote, J., Deluca, A., \& Kosanke, N. (2000). Polysubstance use among alcholics. Journal of Addictive Diseases, 20(4), 57-73.

Tomko, R. L., Baker, N. L., McClure, E. A., et al. (2018). Incremental validity of estimated cannabis grams as a predictor of problems and cannabinoid biomarkers: Evidence from a clinical trial. Drug and Alcohol Dependence, 182, 1-7.

Wray, T. B., Braciszewski, J. M., Zywiak, W. H., \& Stout, R. L. (2016). Examining the reliability of alcohol/drug use and HIV-risk behaviors using Timeline Follow-Back in a pilot sample. Journal of Substance Use, 21(3), 294-297. 
Table 1. Sample characteristics of participants who completed baseline and retest interviews $(n=48)$

\begin{tabular}{lcc}
\hline & Frequency/Mean & $\% /$ SD/range \\
\hline Age & 30.5 & $15.3(S D), 18-64$ (Range) \\
Race: Non-Hispanic Black & 10 & $20.8 \%$ \\
Race: Non-Hispanic White & 24 & $50.0 \%$ \\
Race: Hispanic & 10 & $20.8 \%$ \\
Race: Asian & 3 & $6.3 \%$ \\
Race: American Indian or Alaska Native & 1 & $2.1 \%$ \\
Sex: Female & 17 & $35.4 \%$ \\
Marital status: Never married & 40 & $83.3 \%$ \\
Education: high school or above & 40 & $83.3 \%$ \\
In-person interview & 33 & $68.8 \%$ \\
Zoom interview & 15 & $31.3 \%$ \\
Time between T1 and T2 (in days) & 6.65 & $2.0(S D), 3-11$ (Range) \\
Met criteria for cocaine use disorder in the past & & $52.1 \%$ \\
12 months (conditional on use, $n=48)$ & 25 & $66.7 \%$ \\
Met criteria for alcohol use disorder in the past & & $62.2 \%$ \\
12 months (conditional on use, $n=48)$ & 32 & \\
Met criteria for marijuana use disorder in the & & \\
past 12 months (conditional on use, $n=45)$ & 28 &
\end{tabular}


Table 2. Responses at baseline and retest interviews and test-retest reliability of measured polysubstance use patterns $(n=48)$

\begin{tabular}{|c|c|c|c|c|}
\hline & $\begin{array}{c}\text { Baseline } \\
\text { (mean) }\end{array}$ & $\begin{array}{l}\text { Retest } \\
\text { (mean) }\end{array}$ & ICC & $95 \% \mathrm{CI}$ \\
\hline Number of cocaine (C) use days in past 30 days & 4.60 & 4.10 & 0.94 & $(0.90,0.97)$ \\
\hline $\begin{array}{l}\text { Number of marijuana (M) use days in past } 30 \\
\text { days }\end{array}$ & 15.52 & 15.27 & 0.90 & $(0.83,0.94)$ \\
\hline Number of alcohol (A) use days in past 30 days & 10.44 & 9.42 & 0.90 & $(0.83,0.94)$ \\
\hline Number of CAM days in past 30 days & 1.92 & 1.60 & 0.80 & $(0.67,0.88)$ \\
\hline Number of CA days in past 30 days & 1.17 & 0.83 & 0.47 & $(0.22,0.66)$ \\
\hline Number of CM days in past 30 days & 1.08 & 0.96 & 0.78 & $(0.64,0.87)$ \\
\hline Number of $\mathrm{C}$ only days in past 30 days & 0.44 & 0.71 & 0.42 & $(0.16,0.62)$ \\
\hline Number of A only days in past 30 days & 3.33 & 3.48 & 0.94 & $(0.90,0.97)$ \\
\hline Number of $M$ only days in past 30 days & 8.50 & 9.21 & 0.92 & $(0.87,0.95)$ \\
\hline $\begin{array}{l}\text { Duration of cocaine use session on CAM day } \\
\text { (hours) }^{\text {a }}\end{array}$ & 3.69 & 2.74 & 0.52 & $(0.17,0.75)$ \\
\hline $\begin{array}{l}\text { Duration of alcohol use session on CAM day } \\
\text { (hours) }{ }^{\text {a }}\end{array}$ & 7.72 & 6.43 & 0.73 & $(0.48,0.87)$ \\
\hline $\begin{array}{l}\text { Duration of marijuana use session on CAM day } \\
\text { (hours) }{ }^{\text {a }}\end{array}$ & 3.65 & 3.41 & 0.60 & $(0.28,0.80)$ \\
\hline $\begin{array}{l}\text { Quantity of alcohol use during typical CAM } \\
\text { day (drinks) }{ }^{\text {a }}\end{array}$ & 8.72 & 8.93 & 0.83 & $(0.65,0.92)$ \\
\hline $\begin{array}{l}\text { Quantity of cocaine use during typical CAM } \\
\text { day b }\end{array}$ & $\mathrm{NA}^{\mathrm{d}}$ & $\mathrm{NA}^{\mathrm{d}}$ & 0.26 & $(-0.17,0.61)$ \\
\hline $\begin{array}{l}\text { Quantity of marijuana use during typical CAM } \\
\text { day }{ }^{c}\end{array}$ & $\mathrm{NA}^{\mathrm{d}}$ & $\mathrm{NA}^{\mathrm{d}}$ & 0.33 & $(-0.13,0.67)$ \\
\hline $\begin{array}{l}\text { If access were not an issue, they would } \\
\text { use...(choose all that apply) }\end{array}$ & $\begin{array}{c}\text { Baseline } \\
(n)\end{array}$ & $\begin{array}{c}\text { Retest } \\
(n)\end{array}$ & Kappa & $95 \% \mathrm{CI}$ \\
\hline cocaine & 22 & 24 & 0.67 & $(0.46,0.88)$ \\
\hline alcohol & 23 & 21 & 0.66 & $(0.45,0.88)$ \\
\hline marijuana & 38 & 32 & 0.48 & $(0.22,0.75)$ \\
\hline opioids & 0 & 1 & NA & NA \\
\hline heroin & 1 & 1 & NA & NA \\
\hline sedatives & 1 & 1 & NA & NA \\
\hline stimulants & 4 & 5 & 0.63 & $(0.25,1.00)$ \\
\hline
\end{tabular}

a: $n=24$ conditional on at least one CAM day reported at both baseline and retest.

$b: n=20$ conditional on at least one CAM day reported at both baseline and retest and reported the same units for cocaine use at both baseline and retest.

$\mathrm{c}: \mathrm{n}=18$ conditional on at least one CAM day reported at both baseline and retest and reported the same units for marijuana use at both baseline and retest.

$\mathrm{d}$ : Not Applicable because people could report their use in different units at baseline and retest. 
Table 3. Proportion of reasons for discrepant responses between baseline and retest on pre-selected questions

\begin{tabular}{|c|c|c|c|c|c|c|c|c|c|c|}
\hline & $\begin{array}{c}\text { Q1 } \\
\text { \#CAM } \\
n=48 / 22\end{array}$ & $\begin{array}{c}\mathrm{Q} 2 \\
\# \mathrm{CA} \\
n=47 / 17\end{array}$ & $\begin{array}{c}\text { Q3 } \\
\# \text { CM } \\
n=47 / 13\end{array}$ & $\begin{array}{c}\text { Q4 } \\
\text { s/e CAM } \\
n=24 / 17\end{array}$ & $\begin{array}{c}\text { Q5 } \\
\text { s/e CA } \\
n=14 / 12\end{array}$ & $\begin{array}{c}\text { Q6 } \\
\text { s/e CM } \\
n=5 / 5\end{array}$ & $\begin{array}{c}\text { Q7 } \\
\text { route } \\
\text { CAM } \\
n=24 / 4\end{array}$ & $\begin{array}{c}\text { Q8 } \\
\text { route } \\
\text { CA } \\
n=14 / 2\end{array}$ & $\begin{array}{c}\text { Q9 } \\
\text { route } \\
\text { CM } \\
n=5 / 0\end{array}$ & $\begin{array}{c}\text { Q10 } \\
\text { prefer use } \\
n=48 / 19\end{array}$ \\
\hline $\begin{array}{l}\text { R's situation changed since } \\
\text { the first interview }\end{array}$ & $11(50.0)$ & $7(41.2)$ & $4(30.8)$ & $3(17.6)$ & $2(16.7)$ & 0 & $\begin{array}{c}1 \\
(25.0)\end{array}$ & $3(15.8)$ & NA & $7(36.8)$ \\
\hline $\begin{array}{l}\mathrm{R} \text { didn't remember the } \\
\text { same information at each } \\
\text { interview }\end{array}$ & $9(40.9)$ & $8(47.1)$ & $5(38.5)$ & $5(30.4)$ & $4(33.3)$ & $2(40.0)$ & $\begin{array}{c}1 \\
(25.0)\end{array}$ & 0 & NA & $1(5.3)$ \\
\hline $\mathrm{R}$ reported a different day & $1(4.6)$ & $1(5.9)$ & 0 & $5(29.4)$ & $3(25.0)$ & $1(20.0)$ & 0 & 0 & NA & 0 \\
\hline $\begin{array}{l}\text { The interviewer miscoded } \\
\text { or misunderstood the } \\
\text { response }\end{array}$ & 0 & 0 & 0 & $3(17.6)$ & $2(16.7)$ & 0 & $2(50.0)$ & $1(50.0)$ & NA & $3(15.8)$ \\
\hline $\begin{array}{l}\mathrm{R} \text { didn't understand what } \\
\text { the interviewer was asking }\end{array}$ & 0 & $1(5.9)$ & $1(7.7)$ & 0 & 0 & 0 & 0 & $1(50.0)$ & NA & $3(15.8)$ \\
\hline $\begin{array}{l}\mathrm{R} \text { was not paying attention } \\
\text { on one occasion }\end{array}$ & 0 & 0 & $2(15.4)$ & $1(5.9)$ & 0 & $1(20.0)$ & 0 & 0 & NA & 0 \\
\hline Don't know & $1(4.6)$ & 0 & $1(7.7)$ & 0 & $1(8.3)$ & $1(20.0)$ & 0 & 0 & NA & $1(5.3)$ \\
\hline Other reason & 0 & 0 & & 0 & 0 & 0 & 0 & 0 & NA & $1 *(5.3)$ \\
\hline
\end{tabular}

$n=$ number of non-skipped responses/number of discrepant responses, percentage calculated based on number of discrepant responses

$\mathrm{R}=$ respondent; Q1: Number of CAM days; Q2: Number of CA days; Q3: Number of CM days; Q4: Start and end times of cocaine use on a typical CAM day; Q5: Start and end times of cocaine use on a typical CA day; Q6: Start and end times of cocaine use on a typical CM day; Q7: Route of cocaine use on a typical CAM day; Q8: Route of cocaine use on a typical CA day; Q9: Route of cocaine use on a typical CM day; Q10: If access were not an issue, which drugs would you prefer to use.

Reasons with zero endorsement: $\mathrm{R}$ tried to shorten one of the interviews; $\mathrm{R}$ felt more comfortable in one interview; $\mathrm{R}$ was too embarrassed/thought interviewer would disapprove; $\mathrm{R}$ doesn't really know whether the experience occurred.

* other reason: preference changed 
Figure 1. Test-retest interview flow chart 


\begin{tabular}{|c|c|}
\hline Flow of & \\
\hline $\begin{array}{l}\text { The PSU-SAM starts with a calendar of the past } 30 \text { days. We asked the } \\
\text { participate to mark on the calendar the letter "C" on all the days in the past } \\
30 \text { days when they used cocaine. Then mark "A" on all days they used } \\
\text { alcohol and "M" on all the days they used marijuana. } \\
\text { The program/interviewer check the responses and estimate the number of } \\
\text { days for the following six patterns: } \\
\text { 1. days used cocaine, alcohol and marijuana (CAM) } \\
\text { 2. days used cocaine and alcohol but not marijuana (CA) } \\
\text { 3. days used cocaine and marijuana but not alcohol (CM) } \\
\text { 4. days used cocaine but not alcohol or marijuana (C only) } \\
\text { 5. days drank alcohol but not cocaine or marijuana (A only) } \\
6 . \quad \text { days used marijuana but not cocaine or alcohol (M only) }\end{array}$ & $\begin{array}{l}\text { If days of using CAM>=1, } \\
\text { Q1. The calendar will highlight all [CAM] days and the interviewer ask "You } \\
\text { reported X days when you used [CAM] Think of a typical day starting at } \\
\text { 6:00 AM in the past } 30 \text { days when you used [CAM]. What day was that? } \\
\text { Q2. A vertical timeline representing every hours between 6:00 AM to 5:00 } \\
\text { AM the next day were presented to the respondent, and interviewer ask "On } \\
\text { the timeline, shade all the times you used [cocaine] on that typical day } \\
\text { starting at 6:00 AM when you used [CAM]". } \\
\text { Q3. For each time cocaine were used, the interviewer asked "from [start time } \\
\text { hour] to [end time hour], how much [cocaine] did you used" The respondent } \\
\text { answer a quantity in number and select a correspond unit (like grams, lines, } \\
\text { etc) } \\
\text { Q4. After quantity of use measures, the interviewer ask "What were all the } \\
\text { ways you used cocaine on that typical day when you used CAM?" } \\
\text { Q2-Q4 would be repeated for alcohol (quantity default to standard drink and } \\
\text { Q4 was skipped), then for marijuana } \\
\text { If days of using CAM=0, go to pattern CA }\end{array}$ \\
\hline
\end{tabular}

At the end of PSU-TPS, continue the interview with the SAM, version 5 to measure lifetime and past 12 months substance use and each criteria of substance-specific use disorders.

\section{If days of using $\mathrm{CA}>=\mathbf{1}$}

Repeat Q1-Q4 for cocaine and alcohol. No question related to marijuana use was asked on a CA typical day

\section{If days of using $\mathrm{CA}=\mathbf{0}$, go to pattern $\mathrm{CM}$}

At the end of SAM, continue the interview with the Diagnostic Interview Schedule (DIS), version 5 to measure each criteria of mood and depressive disorders.

\begin{tabular}{ll|} 
At the end of DIS, continue the \\
interview with the Risk Behavior \\
$\begin{array}{l}\text { Assessment (RBA) about other } \\
\text { risk factors associated with } \\
\text { substance use. Then ends the } \\
\text { interview. }\end{array}$ & $\begin{array}{l}\text { At the end of DIS, continue with } \\
\text { the Discrepancy Interview } \\
\text { Protocol (DIP). Then ends the } \\
\text { interview. }\end{array}$ \\
\hline
\end{tabular}

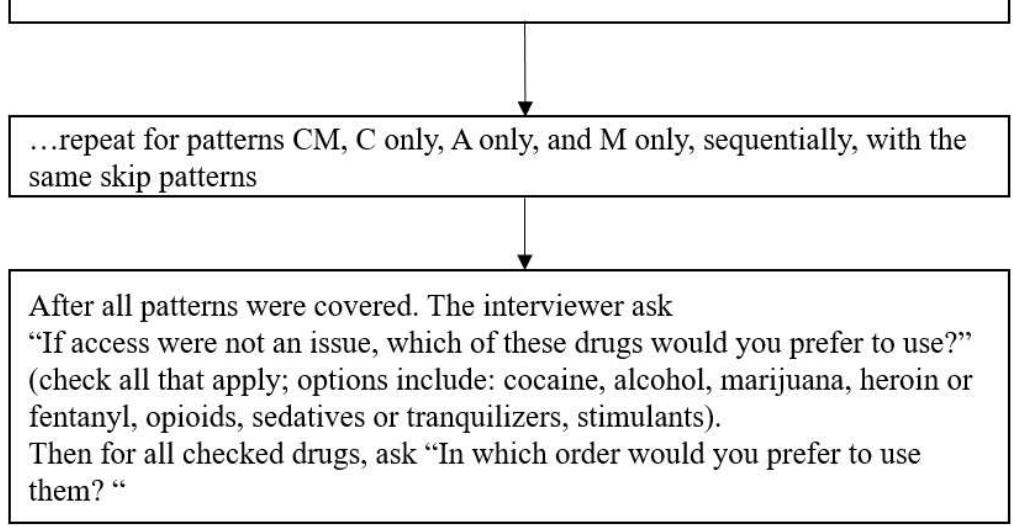

